\title{
Correction to: Gene mutation spectrum and genotype-phenotype correlation in a cohort of Chinese osteogenesis imperfecta patients revealed by targeted next generation sequencing
}

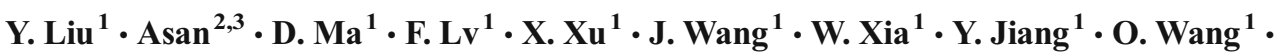

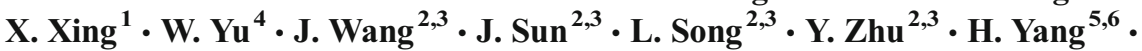 \\ J. Wang ${ }^{5,6} \cdot$ M. Li $^{1}$
}

Published online: 2 November 2017

(C) International Osteoporosis Foundation and National Osteoporosis Foundation 2017

\section{Correction to: Osteoporos Int} https://doi.org/10.1007/s00198-017-4143-8

In Table 2:

Family no. 6 COL1A1 (NM_000088.3) should be c.64313_662delCTATCTTTTCTAGGGTCCCATGGGTC CCCGAGG instead of c.643-13_662delCTATCTTT TCTAGGGTCCCATGGGTCCCC.

Family no. 33 SERPINF1 (NM_002615.5) should be c.271_279dupGCCCTCTCG instead of c.271_279dupGCCCTCT.

The online version of the original article can be found at https://doi.org/ 10.1007/s00198-017-4143-8

\footnotetext{
M. Li

limeilzh@sina.com

Asan

asan@genomics.cn

1 Department of Endocrinology, Key Laboratory of Endocrinology, Ministry of Health, Peking Union Medical College Hospital, Chinese Academy of Medical Science, Beijing 100730, China

2 Tianjin Medical Laboratory, BGI-Tianjin, BGI-Shenzhen, Tianjin 300308, China

3 Binhai Genomics Institute, BGI-Tianjin, Tianjin 300308, China

4 Department of Radiology, Peking Union Medical College Hospital, Chinese Academy of Medical Science, Beijing 100730, China

5 BGI-Shenzhen, Shenzhen 518083, China

6 James D. Watson Institute of Genome Sciences, Hangzhou 310058, China
}

The chromosome translocation mentioned in the final sentence of the second paragraph in the Molecular diagnosis part of the Results section was actually $\mathrm{t}(5 ; 7)(\mathrm{q} 32 ; \mathrm{q} 21)$, not $\mathrm{t}(5 ; 8)(\mathrm{q} 32 ; \mathrm{q} 21)$.

The authors apologize for any inconvenience caused by their errors. 